\title{
Impact of H-mode plasma operation on pre-damaged tungsten divertor tiles in ASDEX
}

\section{Upgrade}

K. Krieger ${ }^{\mathrm{a}, *}$, M. Balden ${ }^{\mathrm{a}}$, B. Böswirth ${ }^{\mathrm{a}}$, J.W. Coenen ${ }^{\mathrm{b}}$, R. Dux ${ }^{\mathrm{a}}$, H. Greuner ${ }^{\mathrm{a}}$, B. Göths ${ }^{\mathrm{b}}$, A. Hakola ${ }^{\mathrm{c}}$, A. Lahtinen ${ }^{\mathrm{c}}$, J. Likonen ${ }^{\mathrm{c}}$, Th. Löwenhoff ${ }^{\mathrm{b}}$, P. de Marne ${ }^{\mathrm{a}}$, G. Pintsuk ${ }^{\mathrm{b}}$, V. Rohde ${ }^{\mathrm{a}}$, G. De Temmerman ${ }^{\mathrm{d}}$, M. Wirtz ${ }^{\mathrm{b}}$ and the ASDEX Upgrade Team ${ }^{\mathrm{a}}$

${ }^{a}$ Max-Planck-Institut für Plasmaphysik, 85748 Garching b. München, Germany

${ }^{b}$ Forschungszentrum Jülich GmbH, Institut für Energie- und Klimaforschung - Plasmaphysik, 52425 Jülich, Germany

${ }^{c}$ VTTechnical Research Centre of Finland, P.O.Box 1000, FIN-02044 VTT, Finland

${ }^{d}$ ITER Organization, Route de Vinon, CS 90 046, 13067 St Paul Lez Durance, France

\begin{abstract}
The effect of continued plasma exposure on two divertor target tiles intentionally damaged before installation was studied for ASDEX Upgrade H-mode discharge conditions.

On one tile, made of molybdenum alloy (TZM), superficial melt damage was created in the GLADIS high heat flux test facility. To measure the influence of the resulting surface corrugations on erosion, the tile surface was subsequently covered with a $20 \mathrm{~nm}$ tungsten marker layer. A second tile, made of tungsten, was pre-damaged by exposure to $2 \times 10^{5}$ consecutive ELM-like heat pulses in the electron beam test facility JUDITH, which resulted in formation of an extended crack network. Both tiles were placed at the outer divertor target of ASDEX Upgrade using the DIM-II divertor manipulator and exposed to a series of 15 identical H-mode discharges. The surface state of both tiles was documented pre- and postexposure using electron scanning microscopy.

SEM analysis of the crack network's microscopic structure did not reveal any additional damage created by plasma exposure but showed deposition of migrated wall materials inside shadowed areas. The erosion pattern of the W-marker layer revealed regions of net erosion as well as of net deposition in the corrugated melt zone. Net erosion up to complete removal of the $\mathrm{W}$ marker layer was found at elevated parts of the surface oriented towards the incident plasma flux whereas net deposition was found in corresponding shadowed areas. In contrast, the undamaged surface parts showed a uniform erosion pattern determined by incident plasma ion flux and temperature.
\end{abstract}




\section{Introduction}

For the plasma facing components (PFCs) of nuclear fusion devices with magnetic plasma confinement tungsten is generally used as material for components subject to high particle and heat loads. A metallic PFC material can, however, become damaged by excessive heat loads, particularly by formation of cracks and by melting. Therefore, strategies need to be developed to assess the lifetime of components providing a set of criteria to decide between replacement of damaged PFCs and continued plasma operation as long as the component's damage stays within an acceptable level [1]. Depending on the characteristic damage processes, thermomechanical properties and performance of affected components will degrade in different ways. On a microscopic scale the plasma facing surface is eroded by sputtering due to the impact of fuel and impurity ions from the plasma. The components are also heated by the power load from the plasma, which may lead to structural damage by recrystallization and crack formation and, at higher loads, may also result in transient melting. Even before the end-of-life criteria are met, the latter processes will damage the plasma exposed surface, which in turn may accelerate the degradation of component performance compared to that expected for a pristine surface structure. Estimates of component life time critically depend on the assumed significance of amplified damage progression by reduced power and particle handling properties of damaged plasma facing surfaces. This is of particular importance for the divertor target components in tokamaks where plasma exhaust leads to the highest power and particle loads.

Generally these effects are studied by power load testing of model PFC systems [2] in ebeam devices [3], neutral beam facilities [4] and plasma guns [5]. These devices cannot, however, fully recreate the component load scenario as encountered in a high flux divertor region with intense plasma flux incident at the target plate under oblique angle of incidence. The experiment presented here was aimed at studying this particular aspect of exposure conditions by exposing divertor target tiles with specific damage types created ex-situ to a series of identical ASDEX Upgrade H-mode plasma discharges. The H-mode scenario used was tailored to have large type-I ELMs. Taking into account the machine size scaling of ELMs [6, 7] their typical energy density at ASDEX Upgrade parameters approximate power load characteristics, which in ITER would correspond to sufficiently mitigated ELMs [8].

\section{Experiment}

For the experiment two tiles with different damage types were prepared. On the first tile, made of molybdenum alloy (TZM), superficial melt damage was created on a circular area of $\approx 40 \mathrm{~mm}$ diameter by exposure in the GLADIS [9] high heat flux test facility. The power load, 
$P=22 \mathrm{MW} / \mathrm{m}^{2}$, and exposure time, $\Delta t=3.51 \mathrm{~s}$, were tailored to create a shallow melt pool for a sufficiently short time to avoid significant melt motion by gravity at the vertically oriented surface (see Figure 1a). To measure the influence of the resulting surface corrugations on erosion, a tungsten marker layer with a thickness of $20 \mathrm{~nm}$ was subsequently deposited on the tile surface by magnetron sputtering.

A second tile, made of tungsten, was pre-damaged in the electron beam test facility JUDITH-2 [10] by exposure to $2 \times 10^{5}$ consecutive ELM-like heat pulses with a characteristic pulse width of $\approx 0.5 \mathrm{~ms}$ and a peak power load of $0.55 \mathrm{GW} / \mathrm{m}^{2}$ similar to what is expected for unmitigated type-I ELMs in ITER [6]. This procedure created an extended crack network within a depth range of $266 \pm 115 \mu \mathrm{m}$ from the surface as shown in Figure 1b) and Figure 1c).

Both tiles were placed at the outer divertor target of ASDEX Upgrade using the DIM-II divertor manipulator [11] and exposed to a series of 15 identical H-mode discharges (discharge numbers 34889-901, 34904-5). Both the exposure geometry and the plasma magnetic configuration are illustrated by Figure 2, which shows a poloidal cross-section of the ASDEX Upgrade vessel in the centre of vessel sector 2 where the divertor manipulator system is installed. Basic discharge parameters were toroidal field $B_{\mathrm{t}}=-2.5 \mathrm{~T}$, plasma current $I_{\mathrm{p}}=0.8 \mathrm{MA}$ and average electron density of $6 \times 10^{19} \mathrm{~m}^{-3}$. The plasma total heating power was $8 \mathrm{MW}$ with $0.5 \mathrm{MW}$ Ohmic heating, $5 \mathrm{MW}$ delivered by neutral beam injection and $2.5 \mathrm{MW}$ by electron cyclotron resonance heating. The ELM frequency in these discharges was $35 \mathrm{~Hz}$ with an average power loss per ELM of $50 \mathrm{~kJ}$. With a total time of $90 \mathrm{~s}$ divertor exposure the target tiles were thus exposed to $\approx 3200$ ELM transients. The outer strike point position was set to the lower edge of the melt area so that the maximum power flux would be delivered to the pre-damaged surface.

The thermal response of the tiles during plasma exposure was observed by an infra-red camera system SCD Cardinal [12] with a frame acquisition rate of $300 \mathrm{~Hz}$. Because the tile image is observed through a glass image guide, the spectral range of that system is restricted to the near-IR with a cut-off towards longer wavelengths at $\approx 2 \mu \mathrm{m}$, which sets a lower limit of $\approx 800^{\circ} \mathrm{C}$ for the detectable temperature range. During the exposures, only a faint signal around the strike point was seen near the end of the discharges. From this result one can conclude that no surface features larger than the spatial resolution of the camera $(\approx 0.5 \mathrm{~mm} / \mathrm{px})$ were heated to temperatures significantly above the detection limit.

In addition to the thermal imaging, the gross erosion flux of molybdenum and tungsten at the Mo tile with tungsten marker layer was measured by spectroscopy in the visible light range. Figure 3 a shows the evolution of the spectral intensity of the tungsten W I line at 
$400.9 \mathrm{~nm}$ over discharges 3-15 of the experiment. The line emission was measured at spectroscopic line of sight (5), which views the tile close to the right edge at $\approx 66 \mathrm{~mm}$ above the outer strike point (see Figure 1a). The measurement shows that $\mathrm{W}$ erosion is strongly correlated to ELMs with a steady decrease of the average level over the entire series of discharges. At the other viewing chords the spectrometer was set to detect the molybdenum Mo I spectral line at $550.6 \mathrm{~nm}$. In contrast to the tungsten signal this line appeared above detection limit in the fourth discharge and its average intensity increased linearly until the end of the experiment. An example is shown in Figure 3b) for the line of sight (1) viewing at $\approx 16 \mathrm{~mm}$ above the OSP (see Figure 1a). From the opposite long term time evolution of the $\mathrm{W}$ and Mo spectral intensities one can infer that the initially uniform $\mathrm{W}$ layer is inhomogeneously eroded so that increasing fractions of the Mo bulk material underneath the $\mathrm{W}$ marker became exposed to the plasma. This conclusion could be confirmed by the postexposure surface analysis, which will be discussed in section 3.

The surface state of both tiles was documented pre- and post-exposure using a Scanning Electron Microscope (SEM) with a dedicated sample manipulation stage for handling of large and heavy specimens up to a mass of $5 \mathrm{~kg}$. To ensure detection of even minor modifications of the surface structure, SEM analysis was performed on representative areas of the tiles at exactly the same locations before and after the experiment. The net erosion/deposition rate was quantified by measuring the thickness of the $\mathrm{W}$-marker in terms of $\mathrm{W}$ areal density preand post-exposure using Rutherford back-scattering spectroscopy (RBS) analysis with $2 \mathrm{MeV}$ ${ }^{4} \mathrm{He}$ ions. This analysis was, however, only providing a spatial resolution of $1.8 \mathrm{~mm}$ given by the diameter of the ${ }^{4} \mathrm{He}$ ion beam. Therefore, the correlation of the lateral distribution of $\mathrm{W}$ layer erosion with surface morphology at microscopic length scales in the sub- $\mu \mathrm{m}$ range was analysed by energy dispersive X-ray analysis (EDX).

\section{Post-exposure analysis}

SEM analysis of the crack network on the tungsten tile after the experiment did not show signs of melt damage created by the plasma exposure. There are, however, detectable modifications of the surface structure due to erosion by plasma impact and deposition of foreign material inside shadowed areas. A representative area at the edge of a surface crack (see Figure 4a) is shown in Figure $4 \mathrm{~b}$ before and after exposure. The arrows in Figure $4 \mathrm{~b}$ indicate surface structures where erosion and deposition respectively are clearly visible after plasma exposure although only on a microscopic scale. EDX analysis of the deposits revealed a mix of predominantly low-Z elements (boron, carbon, oxygen), which is to be expected because the throughput of migrating material is dominated by these elements, even with full 
metal PFCs, owing to the periodic supply of boron by boronisation and of carbon and oxygen from the residual atmosphere and desorption from remote surfaces. Long term growth of these deposits might also contribute to fuel retention. The significance of the retained fuel fraction in comparison to the overall fuel inventory in the plasma facing components cannot be resolved from EDX data alone and requires further experimental investigation by micro-beam Nuclear Reaction Analysis (NRA).

The surface topography of the corrugated melt pool area created on the Mo tile in GLADIS was also studied by SEM imaging before and after exposure. As in the case of the crack network in the pre-damaged tungsten tile, comparison of the pre- and post-exposure surface did not reveal any signs of changes such as melt traces by plasma exposure. RBS analysis of the tungsten marker layer thickness showed, however, strong lateral variations with zones of both net-erosion and net-deposition of tungsten as shown in Figure 5 for a horizontal $\left(\mathrm{RBS}_{\mathrm{H}}\right)$ and vertical $\left(\mathrm{RBS}_{\mathrm{V}}\right)$ line across the corrugated surface. This observation suggested already a correlation of the erosion-deposition pattern with surface topography. The maximum values of both net-erosion and deposition were $\approx 10^{17} \mathrm{~W}$ at $/ \mathrm{cm}^{2}$. Relating this value to an inter-ELM ion fluence of $\approx 10^{20} \mathrm{D}^{+} / \mathrm{cm}^{2}$ derived from Langmuir probe data, one obtains an erosion/deposition fraction of $10^{-3}$. This value sets, however, only an upper limit because intra-ELM measurements of ion saturation current are not reliable due to the insufficent battery voltage of the Langmuir probe diagnostic for high $T_{e}$ during ELMs. Still, the estimate is comparable to previous measurements of tungsten erosion for H-mode conditions $[13,14]$, with tungsten sputtering dominated by low-Z $(\mathrm{B}, \mathrm{C}, \mathrm{O})$ impurity ions at typical plasma concentrations in the 0.1-1 percent range.

The direct correlation of the the lateral distribution of tungsten erosion and deposition from RBS analysis with surface topography is hampered by the low spatial resolution of only $\approx 1.8 \mathrm{~mm}$, given by the diameter of the ${ }^{4} \mathrm{He}$ analysis beam spot. The observed laterally alternating net erosion and deposition zones in Figure 5 represent merely an average over variations on the smaller length scale of the surface corrugation. To overcome this limitation, the $\mathrm{W}$ marker on the Mo tile surface was additionally examined at these smaller length scales by EDX microscopic analysis. A typical example is shown in Figure 6, which covers an area of $3.6 \times 5 \mathrm{~mm}^{2}$ just at the edge of the corrugated melt pool. The location of the analysed area on the tile is shown in Figure 1a labelled as $\mathrm{SEM}_{\mathrm{E}}$. As the tile was installed rotated by $90^{\circ}$ in the SEM, the vertical tile coordinate is along the horizontal axis of the images in Figure 6 and the direction of the incident plasma flux is from top to bottom, indicated by the arrow in image E1. An SEM image of the topography of the analysed area is shown in Figure 6 E1. For even more details two typical areas inside (Figure 6, M1) and outside (Figure 6, D1) the 
corrugated melt zone were examined at tenfold increased magnification. The images E2, M2 and D2 show for the examined areas the results of EDX analysis before plasma exposure. For each image pixel the Mo $\mathrm{L}_{\alpha}$ and $\mathrm{W} \mathrm{M}_{\alpha}$ peak integrals in the corresponding EDX spectrum were determined and pixel colour was set to represent the dominant elemental fraction (red for tungsten and green for molybdenum). Image E2 shows an even distribution of the W layer across the examined area. At larger magnification one observes, however, that at parts of the surface the Mo substrate shines through. This indicates that the magnetron sputter process used to establish the W marker layer did not cover all surface features uniformly. Comparison of the layer structure with the quantitative surface height topography measured by Confocal Laser Scanning Microscopy (CLSM) shows that magnetron deposition was weaker in particular inside groves originating from the tile manufacturing process (image D3). After exposure the identical areas were again analysed by EDX with results shown in images E4, M4 and D4. In line with the ion beam analysis results, one observes that the non-uniform lateral erosion/deposition pattern of the $\mathrm{W}$ marker layer extends to microscopic length scales and is strongly correlated to the surface topography. At the undamaged tile surface area (images D3 and D4) one finds now tungsten being the dominant species inside the manufacturing groves wheras Mo has become fully visible at the leading edges of these grooves indicating complete erosion of the $\mathrm{W}$ layer. From this observation one can infer that tungsten erosion is strongest at leading edges exposed to the strongest local plasma flux as indicated by the arrow in images D3 and D4. In contrast, erosion inside the grooves is much weaker so that re-depositing tungsten ions will collect there. At the surface corrugations in the melt zone (images M3 and M4) this effect is even more pronounced. Comparison of surface height map (M3) and W/Mo surface distribution (M4) shows that the W layer has been completely eroded at the plasma exposed flanks of the two hills in the examined melt area whereas in their shadow tungsten coverage appears to have increased. The sharp separation between tungsten erosion and deposition dominated areas along the ridge line of the hill structure is striking if one takes into account that the slope angles of surface features with typical height in the $10-20 \mu \mathrm{m}$ range are in the same range or lower than the typical ion $\rightarrow$ surface impact angle of $\approx 30^{\circ}$ predicted by ion trajectory simulations [15].

\section{Summary and conclusions}

Two divertor tiles with different surface damage types were exposed to $\approx 90$ s type-I ELM $\mathrm{H}$-mode plasma conditions in the divertor of ASDEX Upgrade. On one tile, made of tungsten, a crack network was created before the experiment by exposure to $2 \times 10^{5}$ ELM-like power transients with a peak power of $0.55 \mathrm{GW} / \mathrm{m}^{2}$ in the JUDITH-2 electron beam facility. On the 
second tile, made of molybdenum, a shallow melt pool was created in the GLADIS heat flux test stand to establish a corrugated re-solidified surface area. For tungsten erosion studies the tile was subsequently covered by a thin $\mathrm{W}$ marker layer.

Comparative analysis of the crack network before and after plasma exposure showed no signs of crack propagation down to lateral dimensions of the order of $100 \mathrm{~nm}$. At this microscopic scale the only effects of plasma exposure were minor erosion of plasma-exposed crack edges and deposition of migrating non-volatile plasma impurities in shadowed areas, particularly inside the cracks. Co-deposition with these layers might contribute to the overall fuel retention although the significance of this effect could not be clarified by the employed analysis methods.

As in the case of the crack network no signs of further melt damage were observed at the melt zone of the Mo tile down to the same lateral dimensions of $\mathrm{O}(100 \mathrm{~nm})$. Analysis of the $\mathrm{W}$ marker layer revealed that $\mathrm{W}$ local net erosion and deposition respectively are strongly correlated with surface topography. It turned out that tungsten is predominantly eroded at plasma facing flanks of surface features whereas in shadowed areas $\mathrm{W}$ re-deposition appears to prevail over erosion leading to net deposition of material. However, for the undamaged surface parts erosion appeared uniform at length scales much larger than surface roughness features from the manufacturing process.

The experiment showed no detrimental effects of the damaged target plate areas on plasma operation. One can further conclude that fast damage progression by sustained plasma operation on superficially damaged PFCs on a time scale of several 10 s of seconds is not to be expected for the comparatively small type-I ELM transients in medium sized tokamaks such as ASDEX Upgrade. The result is still of relevance for the plasma operational space of large devices such as ITER because material limits in these devices do not allow for ELM energy densities significantly exceeding the values typical for large type-I ELMs in ASDEX Upgrade. Extrapolating the outcome of the experiment to plasma operation on a much longer time scale and/or higher transient energy densities will require a two-fold approach. Firstly, the degradation of plasma facing materials at extreme plasma fluence levels and extreme repetition numbers of power transients requires further studies in linear plasma devices and tokamaks with quasi-stationary plasma discharges at ITER-relevant divertor power flux level. In contrast to usual component qualification procedures, however, where tests end once a predefined damage pattern has emerged, future studies should, for established component designs, continue exposure until complete failure of the components, such as by macroscopic cracking or disintegration. In a second step these studies should be complimented by investigation of plasma response to heavily damaged components before ultimate failure to 
derive suitable end-of-life criteria for a component in terms of mandatory replacement of the component before further plasma operation is possible. The obtained data set will then in turn provide the basis for validation of predictive modelling of effects such as increased impurity sources from damaged surfaces for extrapolation to large devices.

\section{Acknowledgement}

This work has been carried out within the framework of the EUROfusion Consortium and has received funding from the Euratom research and training programme 2014 - 2018 and 2019 - 2020 under grant agreement No 633053. The views and opinions expressed herein do not necessarily reflect those of the European Commission. 


\section{References}

[1] Y. Ueda, K. Schmid, M. Balden, J.W. Coenen, T. Loewenhoff, A. Ito, A. Hasegawa, C. Hardie, M. Porton, M. Gilbert, Baseline high heat flux and plasma facing materials for fusion, Nucl Fusion 57(9) (2017).

[2] T. Hirai, F. Escourbiac, S. Carpentier-Chouchana, A. Durocher, A. Fedosov, L. Ferrand, T. Jokinen, V. Komarov, M. Merola, R. Mitteau, R.A. Pitts, W. Shu, M. Sugihara, V. Barabash, V. Kuznetsov, B. Riccardi, S. Suzuki, ITER full tungsten divertor qualification program and progress, Phys Scripta T159 (2014) 5.

[3] M. Wirtz, A. Kreter, J. Linke, T. Loewenhoff, G. Pintsuk, G. Sergienko, I. Steudel, B. Unterberg, E. Wessel, High pulse number thermal shock tests on tungsten with steady state particle background, Phys Scripta T170 (2017) 8.

[4] H. Greuner, B. Böswirth, T.R. Barrett, F. Crescenzi, F. Gallay, K. Hunger, M. Richou, E. Visca, A. von Müller, J.H. You, Progress in high heat flux testing of European DEMO divertor mock-ups, Fusion Eng Des (2018) accepted for publication.

[5] N. Klimov, V. Podkovyrov, A. Zhitlukhin, D. Kovalenko, B. Bazylev, G. Janeschitz, I. Landman, S. Pestchanyi, G. Federici, A. Loarte, M. Merola, J. Linke, T. Hirai, J. Compan, Experimental study of PFCs erosion under ITER-like transient loads at plasma gun facility QSPA, J Nucl Mater 390-91 (2009) 721-726.

[6] T. Eich, B. Sieglin, A.J. Thornton, M. Faitsch, A. Kirk, A. Herrmann, W. Suttrop, J.E.T. Contributors, M.S.T. Contributors, U. Asdex, M. Teams, ELM divertor peak energy fluence scaling to ITER with data from JET, MAST and ASDEX Upgrade, Nuclear Materials and Energy 12 (2017) 84-90.

[7] B. Sieglin, M. Faitsch, T. Eich, A. Herrmann, W. Suttrop, J.E.T. Collaborators, M.S.T. Team, A.U. Team, Progress in extrapolating divertor heat fluxes towards large fusion devices, Phys Scripta T170 (2017) 6.

[8] R.A. Pitts, S. Carpentier, F. Escourbiac, T. Hirai, V. Komarov, S. Lisgo, A.S. Kukushkin, A. Loarte, M. Merola, A.S. Naik, R. Mitteau, M. Sugihara, B. Bazylev, P.C. Stangeby, A full tungsten divertor for ITER: Physics issues and design status, J Nucl Mater 438 (2013) S48S56.

[9] H. Greuner, B. Boeswirth, J. Boscary, P. McNeely, High heat flux facility GLADIS: Operational characteristics and results of W7-X pre-series target tests, J Nucl Mater 367 (2007) 1444-1448.

[10] P. Majerus, R. Duwe, T. Hirai, W. Kuhnlein, J. Linke, M. Rodig, The new electron beam test facility JUDITH II for high heat flux experiments on plasma facing components, Fusion Eng Des 75-79 (2005) 365-369.

[11] A. Herrmann, N. Jaksic, P. Leitenstern, H. Greuner, K. Krieger, P. de Marne, M. Oberkofler, V. Rohde, G. Schall, A.U. Team, A large divertor manipulator for ASDEX Upgrade, Fusion Eng Des 98-99 (2015) 1496-1499. 
[12] P.C. Klipstein, E. Avnon, Y. Benny, R. Fraenkel, A. Glozman, S. Grossman, O. Klin, L. Langoff, Y. Livneh, I. Lukomsky, M. Nitzani, L. Shkedy, I. Shtrichman, N. Snapi, A. Tuito, E. Weiss, InAs/GaSb Type II superlattice barrier devices with a low dark current and a high quantum efficiency, in: B.F. Andresen, G.F. Fulop, C.M. Hanson, P.R. Norton (Eds.), Infrared Technology and Applications X1, Spie-Int Soc Optical Engineering, Bellingham, 2014.

[13] A. Thoma, K. Asmussen, R. Dux, K. Krieger, A. Herrmann, B. Napiontek, R. Neu, J. Steinbrink, M. Weinlich, U. Wenzel, Spectroscopic measurements of tungsten erosion in the ASDEX Upgrade divertor, Plasma Phys Contr F 39(9) (1997) 1487-1499.

[14] R. Dux, V. Bobkov, A. Herrmann, A. Janzer, A. Kallenbach, R. Neu, M. Mayer, H.W. Muller, R. Pugno, T. Putterich, V. Rohde, A.C.C. Sips, A.U. Team, Plasma-wall interaction and plasma behaviour in the non-boronised all tungsten ASDEX Upgrade, J Nucl Mater 39091 (2009) 858-863.

[15] R. Chodura, NUMERICAL-ANALYSIS OF PLASMA WALL INTERACTION FOR AN OBLIQUE MAGNETIC-FIELD, J Nucl Mater 111(NOV-) (1982) 420-423. 


\section{Figures}

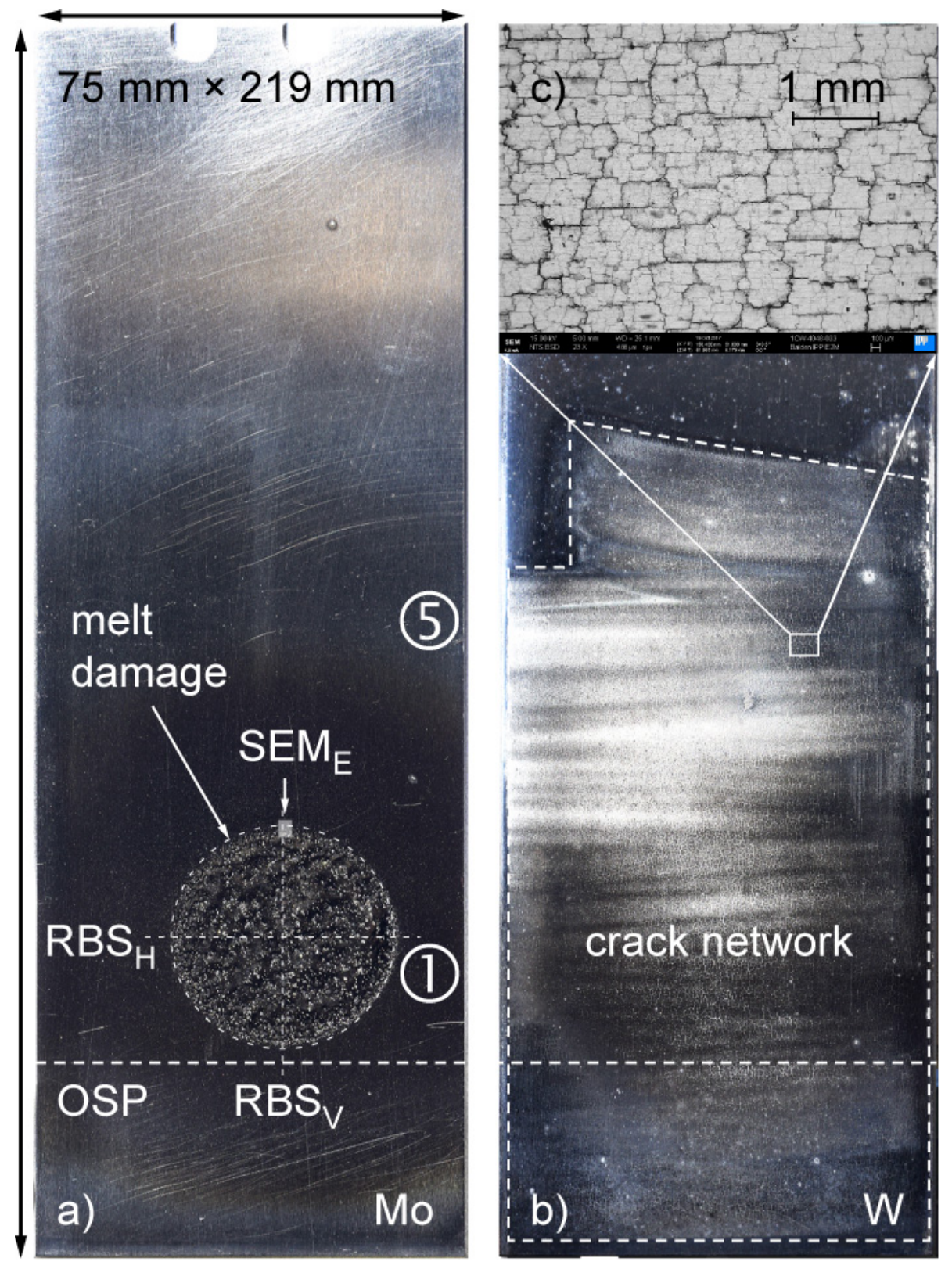

Figure 1: a) Mo tile with melt damage area and $20 \mathrm{~nm} \mathrm{~W}$ marker layer. Labels (1) and (5) denote positions of corresponding spectroscopic viewing chords. Labels $S E M_{E}, R B S_{H}$ and $R B S_{V}$ indicate locations of microscopic and ion beam analysis as discussed in the text. $\mathrm{b}$ ) Tungsten tile with crack network area indicated by dashed contour line. c) Magnified view of crack network surface. The horizontal dashed line represents the position of the outer strike point (OSP) during plasma flat-top. 


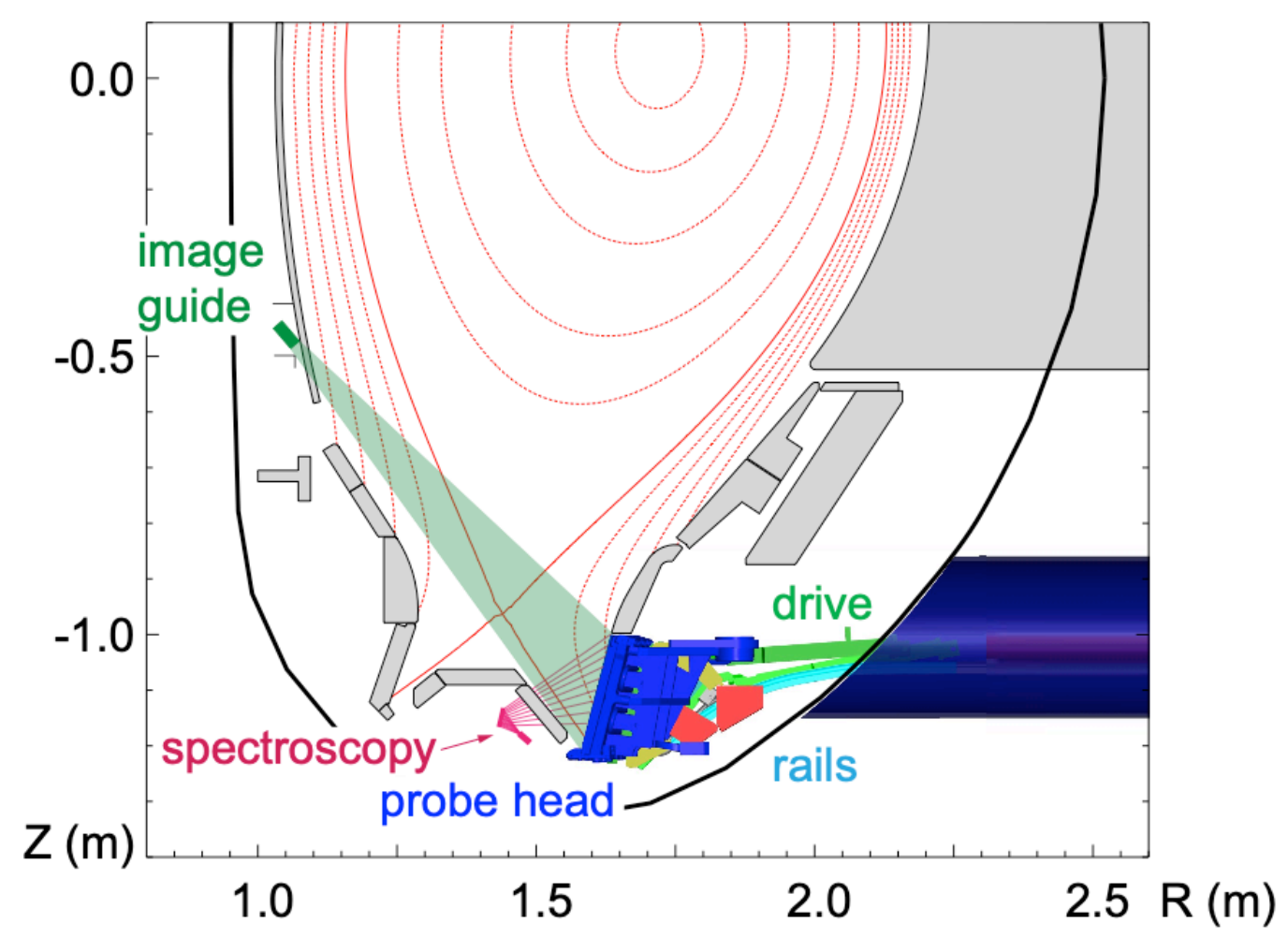

Figure 2: Cross-section of the ASDEX Upgrade vessel with the probe head of the divertor manipulator in exposure position at the outer divertor target. Also shown are the observation geometries of the local visible range spectroscopy and of the image guide optics of the nearinfra-red camera. Flux surfaces are plotted for the flat-top phase of discharge 34892 at $t=4.0 \mathrm{~s}$ 

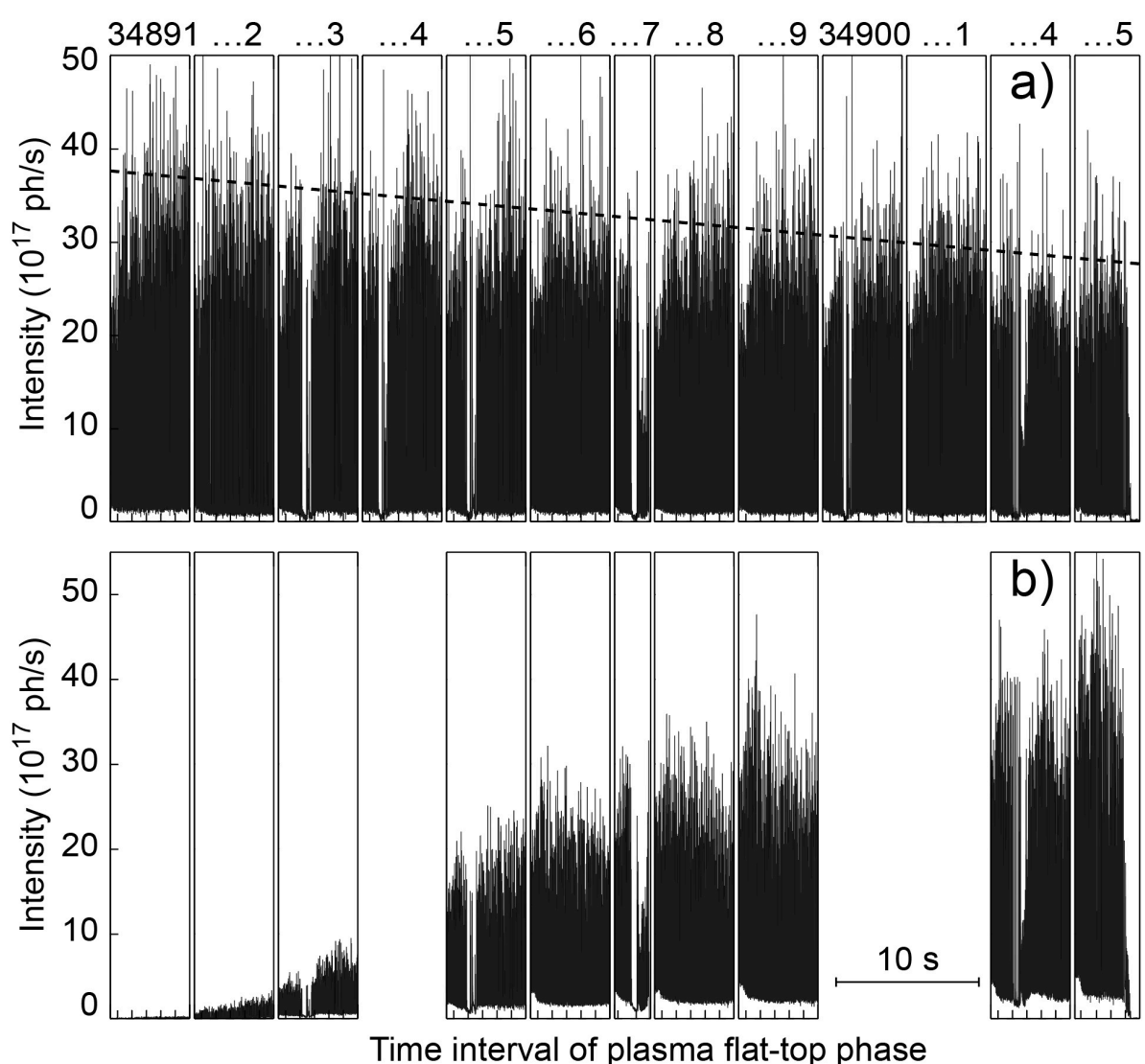

Figure 3: Time evolution of the spectral intensity of the tungsten W I emission line at $400.9 \mathrm{~nm}$ on the tungsten marker coating $66 \mathrm{~mm}$ above the outer strike point (a) and spectral intensity of the molybdenum Mo I emission line at $550.6 \mathrm{~nm} 16 \mathrm{~mm}$ above the outer strike point (b). 

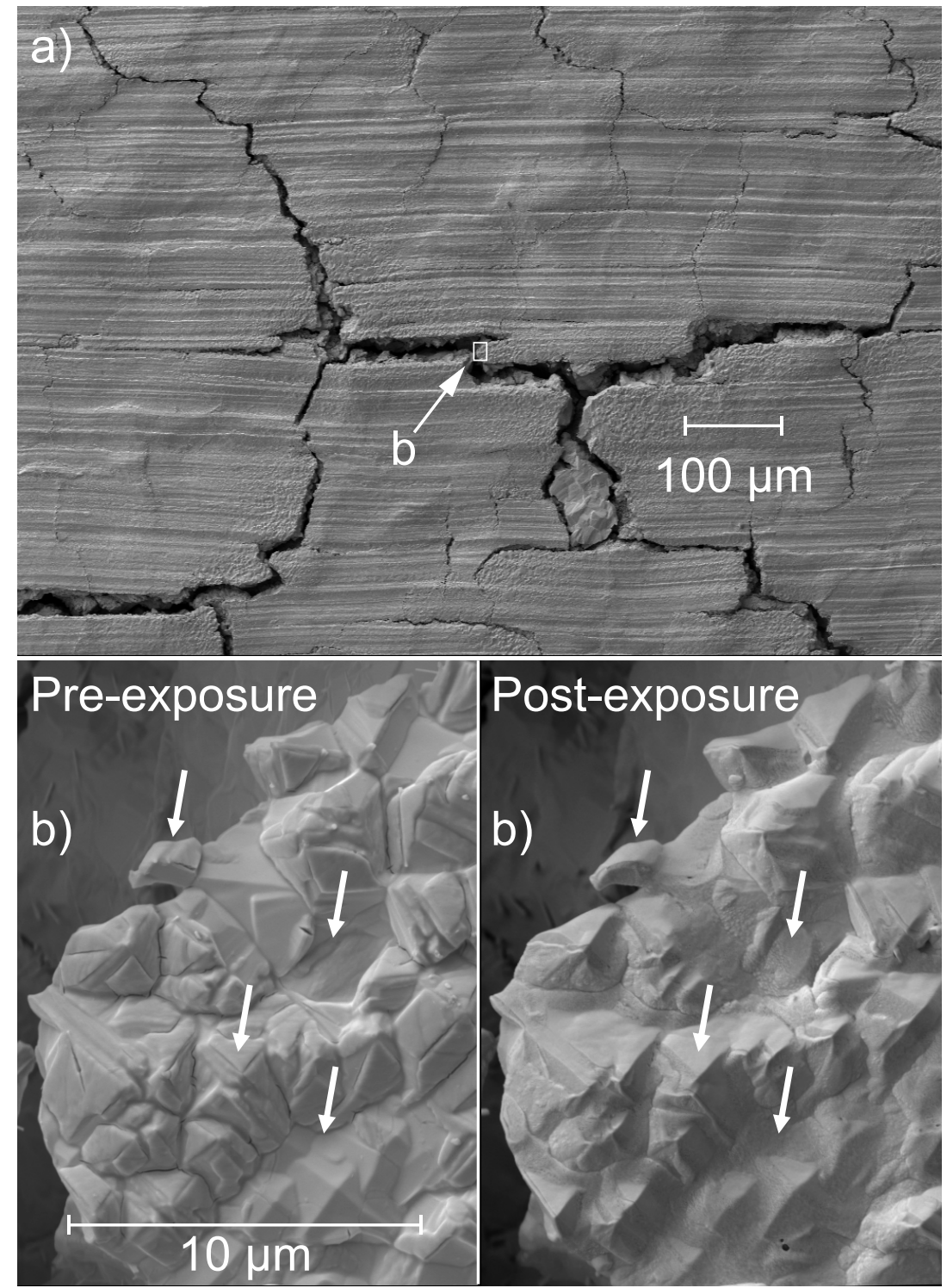

Figure 4: a) SEM image of a typical crack pattern at the tungsten tile with e-beam ELM-like loading. b) Magnified area at the edge of a crack before and after plasma exposure. The arrows in the post-exposure image denote typical erosion and deposition patterns. 

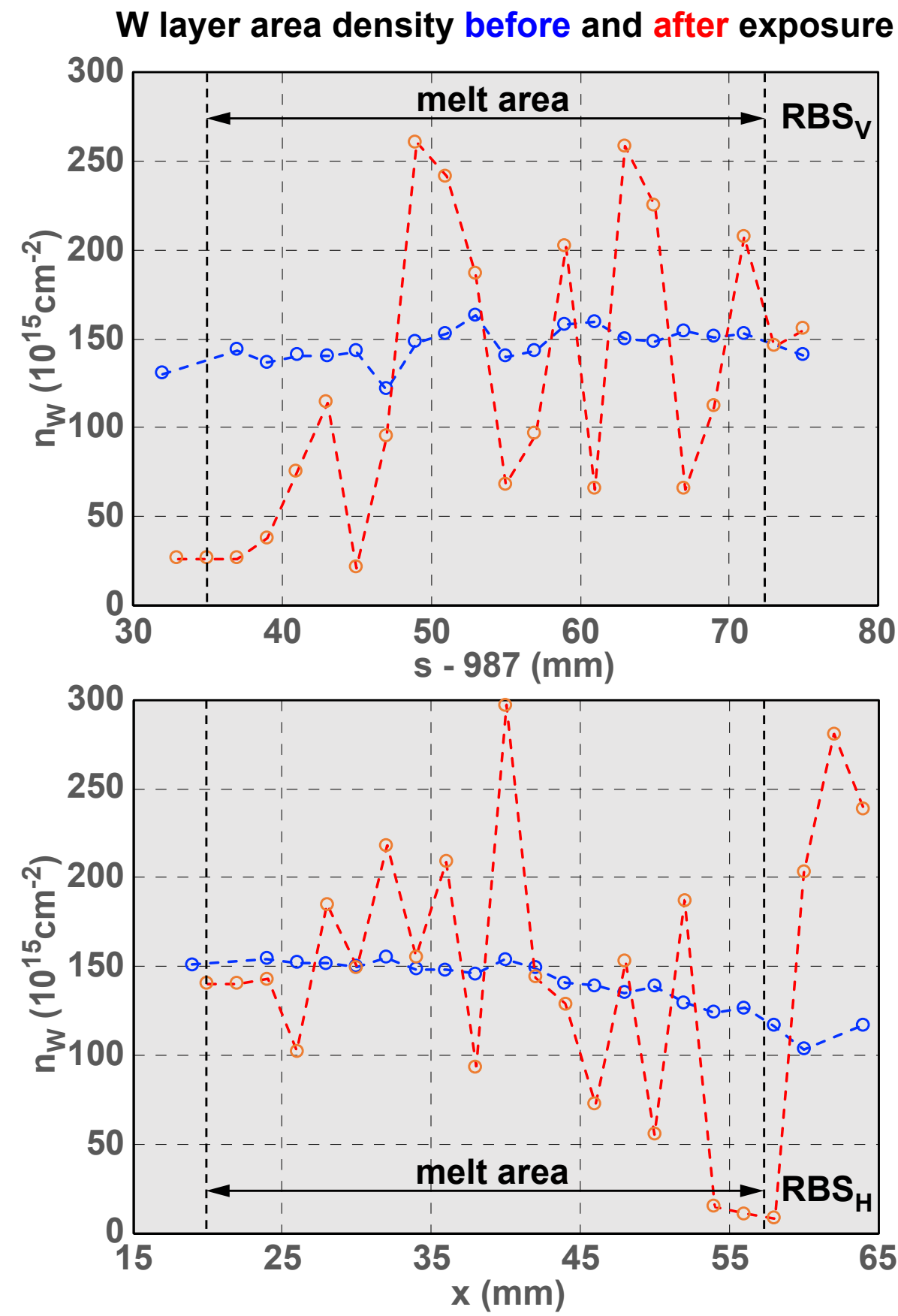

Figure 5: Thickness of W marker layer across melt zone at Mo tile measured by RBS along a line in poloidal (vertical on tile) direction $\left(\mathrm{RBS}_{\mathrm{V}}\right)$ and a line in toroidal (horizontal on tile) direction $\left(\mathrm{RBS}_{\mathrm{H}}\right)$. 

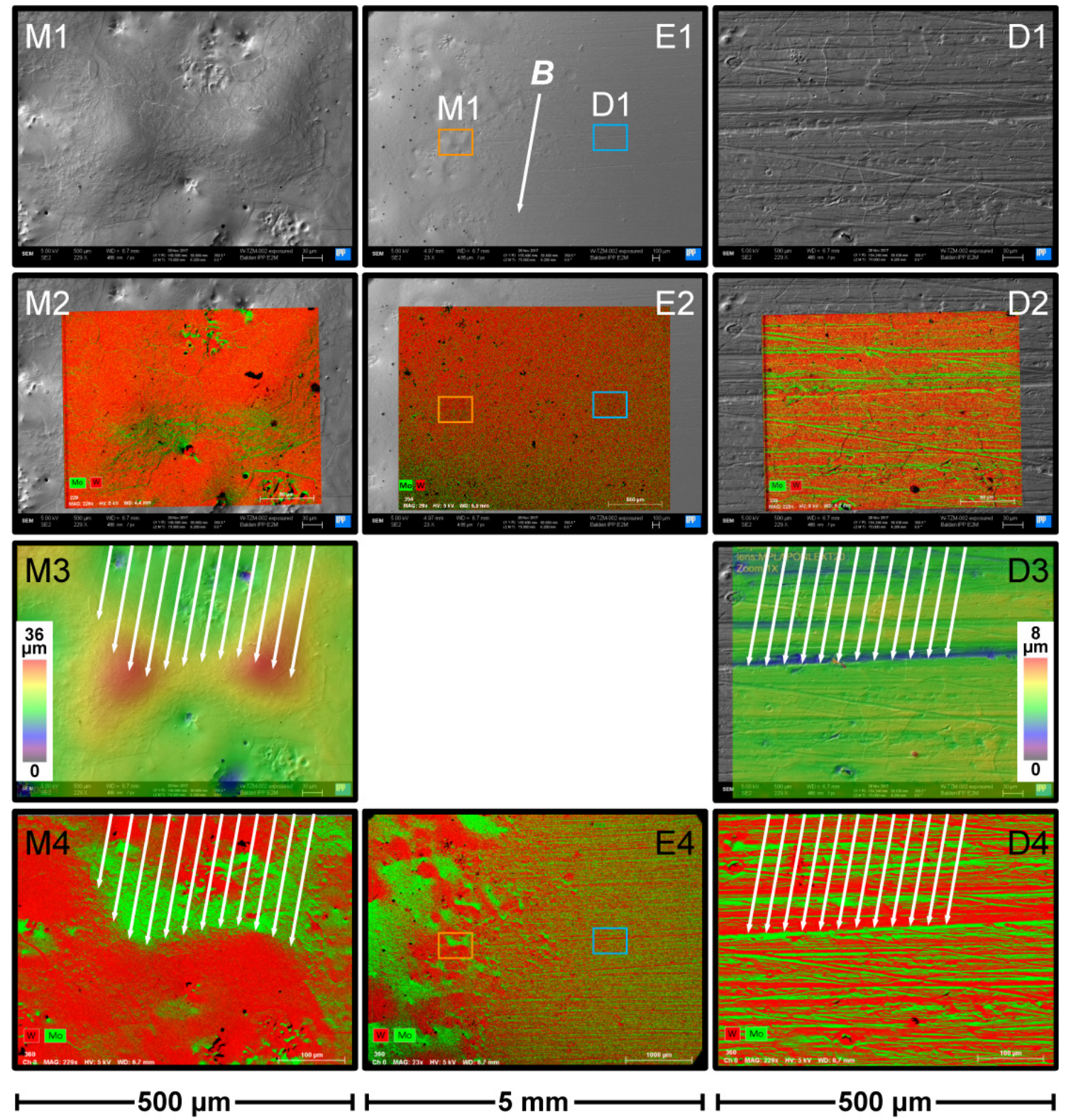

$5 \mathrm{~mm}$

$500 \mu \mathrm{m}$

Figure 6: Surface analysis of the melt area on the Mo tile. For analysis the tile was installed in the SEM rotated by $90^{\circ}$ to its orientation in the divertor. SEM image E1 shows a magnified view of the region with label SEME in Figure 1. Detailed views at ten-fold increased magnification are shown for a typical area inside the melt zone (M1) and at the default tile surface (D1). The white arrow indicates the direction of the orientation of the magnetic field and the direction of the incident plasma flux. Images E2, M2 and D2 show results of EDX analysis before exposure with pixel colours showing the respective strongest elemental concentration (red for tungsten and green for molybdenum). Images E4, M4 and D4 show corresponding results after exposure and images M3 and D3 show height maps of the respective surface areas obtained by CLSM. 